\title{
TIC y discapacidad. Conocimiento del profesorado de educación primaria en Andalucía
}

\author{
José Mª Fernández Batanero*, Pedro Román Graván*, Mohammed El Homrani** \\ *Universidad de Sevilla, **Universidad de Granada
}

\section{RESUMEN}

En la actualidad, debemos considerar la formación permanente del profesorado y, con ella, el aprendizaje continuo, como necesarios y esenciales para desarrollar una educación de calidad en igualdad y equidad. En este contexto, las TIC abren una nueva forma de acceder a la información y un gran puente de comunicación, ya que para algunas personas las tecnologías constituyen la única vía de acceso al mundo educativo y de la cultura. En este artículo se presentan los resultados obtenidos en una investigación cuyo propósito se centró en la identificación del nivel de formación y conocimiento tecnológico que el profesorado de educación primaria, de las provincias de Granada, Huelva, Jaén y Sevilla, tienen respecto a la aplicación de las TIC para personas con discapacidad. A partir de un diseño de investigación del tipo ex post-facto descriptivo, la muestra estuvo compuesta por 425 docentes. Como estrategia de recogida de información empleamos un cuestionario confeccionado ad hoc, validado mediante el procedimiento de "Coeficiente de competencia experta" o "Coeficiente K". El nivel de fiabilidad del instrumento fue analizado mediante la alfa de Cronbach. Entre los resultados obtenidos destacamos la baja capacitación del profesorado.

Palabras Clave: tecnologías de la información y comunicación, discapacidad, inclusión educativa, accesibilidad.

\section{ICT and disability. Knowledge of the teachers of primary education in Andalusia}

\section{ABSTRACT}

At present, we must consider the ongoing training of teachers and, with it, continuous learning, as necessary and essential to develop a quality education in equality and equity. In this context, ICTs open a new way of accessing information and a great communication bridge, since for some people technologies are the only way to access the world of education and culture.

The article presents the results of an investigation whose purpose is focused on identifying the level of training and technological knowledge that the specialist in physical education primary teachers of Primary Education of Seville and its province, with respect to the application of ICT for people with disabilities. From a research design type descriptive ex post facto, the sample was composed of 87 teachers. As information collection strategy used a questionnaire prepared ad hoc, validated by the process of "coefficient expert competence" or "K Coefficient". The level of reliability of the instrument was analyzed by Cronbach's alpha. Among the results we highlight the low training of primary school teachers regarding the application of ICT for students with disabilities.

Keywords: information and communication technology, disability, educational inclusion, accessibility.

\section{Agradecimientos}

Esta investigación ha sido realizada con el apoyo del Ministerio de Economía y Competitividad Español, en el marco del Plan Estatal de Fomento de la Investigación Científica y Técnica de Excelencia 2013-2016 (proyecto DIFOTICYD EDU2016 75232-P).

\section{Introducción}

En la última década, han sido muchos los organismos internacionales que, a través de los estudios que han promovido, vienen reclamando la necesidad de pasar de la retórica de los principios a la realidad de los hechos. Ahora bien, dicha realidad no sería posible sin tener en cuenta el apoyo educativo que prestan las Tecnologías de la Información y Comunicación (TIC) al proceso inclusivo, puesto que constituyen el andamiaje que va a permitir realizar tareas ajustadas a las posibilidades e intereses de las personas.

La inclusión de las TIC en el currículo es un tema complejo ya que se requiere formación específica que implica la habilitación para aprovechar las potencialidades de las TIC para la gestión docente, su desarrollo profesional y el aprendizaje a lo largo de la vida. Ahora bien, dicha formación toma más relevancia en las aulas ante la presencia de alumnado con diversidad funcional 
por discapacidad. En este sentido, la interacción entre las TIC y la diversidad funcional por discapacidad, es decir, las formas en las que éstas puede contribuir al desarrollo de ambientes de aprendizaje que tengan en cuenta a esta diversidad de alumnado, bajo los principios de calidad, igualdad de oportunidades y equidad, suponen un factor educativo de primer orden y una línea prioritaria de investigación. En esta línea, podemos decir que son numerosos los autores que señalan que el acceso a las TIC ofrece un alto nivel igualador de oportunidades a las personas (García García y López Azuaga, 2012; Marín, 2013; Cabero, Fernández Batanero y Córdoba, 2016).

\section{Estado actual}

En el ámbito internacional son abundantes los estudios que resaltan la importancia de la integración de la tecnología para la mejora del aprendizaje de "todo" el alumnado (Ghaleb, 2014; Khetarpal, 2015; Alper y Goggin, 2017), pero son más escasos aquellos que realizan especial hincapié en el alumnado con necesidades educativas especiales por discapacidad (Wallace y Georgina, 2014; Istenic y Bagon, 2014). Se pone de manifiesto el potencial que tienen las TIC para contribuir a una mejor calidad de vida en los estudiantes con diversidad funcional, siendo una prueba de ello los diferentes estudios que se han realizado al respecto en los últimos años (Patton y Roschelle, 2008; Bouck, Doughty, Flanagan, Szwed y Bassette, 2010). También, aunque muy escasos, se han realizando estudios donde se destaca la falta de formación del profesorado para enseñar con éxito las TIC, en el marco de la educación especial (Liu, 2011; Yusof, Gnanamalar, Low, y Aziz, 2014; Altinay y Altinay, 2015; Vladimirovna y Sergeevna, 2015).

En el contexto español, a nivel general, los estudios realizados respecto a la capacitación de los docentes para el manejo de las TIC apuntan que estos tienen altas actitudes hacia ellas, pero se sienten inseguros para su incorporación a los proceso de enseñanza-aprendizaje, y no tanto desde un punto de vista tecnológico, sino más bien desde una perspectiva didáctica y metodológica (Suárez, Almerich, Gallardo y Aliaga, 2013; Prendes y Gutiérrez, 2013; Valdivieso y González, 2016). Ello explica la poca variabilidad de materiales tecnológicos que el profesorado utiliza con el alumnado en su actividad profesional (Ferrandis, Grau y Fortes, 2010).

En el caso de su capacitación para la utilización de las TIC aplicadas a sujetos con diversidad funcional por discapacidad, lo primero a señalar es la escasez de trabajos existentes, como puede observarse en aquellos estudios que abordan la problemática de la capacitación en TIC de los profesores y las competencias que necesitan para utilizarlas con personas con necesidades educativas especiales, donde para nada se aborda la temática de las TIC y la diversidad funcional (Suriá, Martínez y Ordoñez, 2010; Rosario y Vázquez, 2012; Terigi, 2013; Rangel y Peñalosa, 2013; Ortiz, Almazán, Peñaherrera y Cachón, 2014). Por otra parte, apuntar que en los pocos trabajos realizados, se señala la falta de formación y conocimiento que tiene el profesorado respecto a los diferentes tipos de tecnologías que pueden utilizarse como recursos de apoyo a las personas con discapacidad, así como las posibilidades que ofrecen y las funciones para las que pueden ser utilizadas (Roig, Ferrández, Rodríguez-Cano y Crespo, 2012; Tello y Cascales, 2015).

Dicha formación se hace más necesario al tener en cuenta que últimamente se ha avanzado bastante al analizar las TIC como instrumentos significativos que favorecen la inclusión de las personas con diferentes tipos de diversidad cognitivas, sensoriales, o motóricas, y que pueden ayudar a superar las limitaciones que se derivan de las mismas (Homero, Tejedor y Calvo, 2017). En concreto pueden favorecer la autonomía de los estudiantes, ya que se pueden adaptar a las necesidades y demandas de cada alumno o alumna de forma más personal; ofrecer un feed-back inmediato; favorecer la comunicación sincrónica y asincrónica de estos estudiantes con el resto de compañeros y el profesorado; ahorrar tiempo para la adquisición de habilidades y capacidades; favorecer el diagnóstico del estudiante; respaldar un modelo de comunicación y de formación multisensorial; propiciar una formación individualizada, ya que los alumnos puedan avanzar a su propio ritmo, lo cual es de extremada importancia para estos sujetos; favorecer el desarrollo de la autonomía e independencia de las personas; evitar la marginación, la brecha digital, que introduce el verse desprovisto de utilizar las herramientas de desarrollo de la sociedad del conocimiento; facilitar la inserción sociolaboral del alumno con dificultades específicas; proporcionar momentos de ocio; ahorrar tiempo para la adquisición de habilidades y destrezas; los ejercicios que deben realizar los alumnos pueden ser ejecutados y repetidos con mínimos esfuerzos para que los estudiantes adquieran las competencias, actitudes y capacidades; propician el acercamiento de estas personas al mundo científico y cultural; o que pueden ser excelente simuladores (Toledo, 2013).

No debemos olvidar el fuerte volumen de TIC que progresivamente va apareciendo para alumnado con necesidades educativas de apoyo específico, en los diferentes niveles educativos, y que requiere que desde los centros de formación y los respectivos Centros de Profesorado (CEP) se realicen esfuerzos específicos para que los docentes actuales y los futuros las conozcan, y que al mismo tiempo se encuentren capacitados para su utilización.

\section{Planteamiento del problema y justificación del estudio}

El factor más crítico para el desarrollo de la educación inclusiva es el profesorado. Todavía son muchos los docentes de educación primaria que no se encuentran suficientemente cualificados para afrontar la profunda transformación que requiere el sistema educativo, bajo el reto que supone una sociedad altamente digitalizada. Los esfuerzos por mejorar las competencias docentes de los profesionales vinculados con ella, tienen que estar presididos por una comprensión de los factores que facilitan el desarrollo de buenas prácticas educativas de orientación inclusiva con TIC.

En este sentido, nuestra pretensión se basa en el establecimiento de una relación entre el conocimiento que tiene el profesorado sobre las "TIC" y su aplicación para personas con diversidad funcional por "discapacidad", no sólo como elemento facilitador de calidad, sino también como factor de inclusión educativa. Así pues, las preguntas que nos hacemos son: ¿Qué formación y conocimiento tecnológico tienen los maestros de educación primaria, de las provincias andaluzas de Granada, Huelva, Jaén y Sevilla (España), en relación con las personas con discapacidad? ¿Este conocimiento está determinado por variables como el género, años de experiencia o el tipo de centro educativo donde imparten su docencia? ¿El nivel de formación y conocimiento tecnológico, respecto a la aplicación de las TIC en personas con discapacidad es igual o diferente en función de los tipos de diversidad: auditiva, motora, visual o cognitiva?

Las razones esenciales que justifican este estudio han sido básicamente tres: En primer lugar, su relevancia, ya que las TIC constituyen herramientas indispensables para la producción y transmisión de conocimientos, la generación de riqueza y en general, para el desarrollo. Esta condición es aplicable para todos los grupos de población y particularmente para las personas que ven disminuidas sus posibilidades por alguna situación de 
discapacidad física, cognitiva o sensorial. En segundo lugar, su novedad, ya que existen escasos estudios en nuestro país que hayan desarrollado este tipo de trabajos en contextos reales. $\mathrm{Y}$ por último, su demanda internacional, puesto que los organismos europeos e internacionales (UNESCO, AEDNEE "Agencia Europea para el Desarrollo de las Necesidades Educativas Especiales", OCDE, entre otros) reclaman de los países, cada vez con más urgencia, la puesta en marcha de programas que favorezcan la inclusión educativa, haciendo especial referencia a las personas con diversidad funcional.

\section{Método}

Nuestro estudio tiene como objetivo principal conocer el nivel de formación y conocimiento tecnológico que los maestros de educación primaria, de las provincias andaluzas de Granada, Huelva, Jaén y Sevilla (España), tienen respecto a la aplicación de las TIC para personas con diferentes tipos de discapacidades. Más concretamente la exploración se concretó en los siguientes objetivos específicos:

a) Conocer si el nivel de formación y conocimiento tecnológico, está determinado por variables como el género, años de experiencia o el tipo de centro educativo donde imparten su docencia (Público, Privado o Privado-concertado).

b) Conocer si el nivel de formación y conocimiento tecnológico, respecto a la aplicación de las TIC en personas con discapacidad es igual o diferente en función de los tipos de diversidad: auditiva, motora, visual, cognitiva,...

\section{Diseño y muestra}

Para dar respuesta al objetivo planteado, optamos por un diseño de investigación del tipo ex post-facto descriptivo, utilizándose un muestreo no probabilístico causal o accidental. Así pues, la muestra estuvo compuesta por 425 profesores y profesoras de educación primaria de las provincias de Granada, Huelva, Jaén y Sevilla (España). Su distribución es la siguiente (Tabla 1):

Tabla 1. Distribución profesorado por provincias.

\begin{tabular}{lcc} 
Provincia & $\mathrm{n}^{\mathbf{o}}$ de profesores & $\%$ \\
\hline Granada & 113 & 26,5 \\
Huelva & 98 & 23 \\
Jaén & 90 & 21,1 \\
Sevilla & 124 & 29,1 \\
\hline
\end{tabular}

De ellos 59 (67,8\%) eran mujeres y 28 (32,1\%) hombres. Indicar que el $26,4 \%$ ( $\mathrm{f}=112)$ impartían primer ciclo de primaria, el 33,6\% ( $f=143$ ) segundo ciclo y el $40 \%$ ( $f=170)$ tercer ciclo de primaria. En relación a la variable "tipo de centro" decir que la gran mayoría pertenecen a centros educativos públicos $(65,4 \%)$, seguidos por los centros privados-concertados (30\%) y los de carácter privado $(4,6 \%)$.

\section{Instrumento de evaluación}

Para la recogida de información se utilizó un cuestionario elaborado ad hoc a partir de la revisión de la literatura como caracterización de TIC_(Ortíz, Almanzán, Peñaherrera y Cachón, 2014; Rangel, 2015, etc.) y necesidades educativas especiales (Cabero, Fernández Batanero y Córdoba, 2016). El cuestionario final denominado "DIFOTICyD" (Diagnóstico y formación del profesorado para la incorporación de las TIC en alumnado con diversidad funcional), estaba conformado por 53 ítems agrupados en 6 dimensiones (General, visual, auditivo, motórico, cognitivo y accesibilidad). Para cada una de estas declaraciones se solicitaba al profesorado que marcaran la importancia concedida sobre una escala de diez puntos $(0=$ Nada desarrollada, 2 y 3: Muy Poco desarrollada, 4 y 5: Poco desarrollada, 6 y 7: Algo Desarrollada, 8 y 9: Bastante desarrollada, 10=Muy desarrollada). El instrumento se administró vía Internet, al comienzo del primer cuatrimestre del curso académico 2016-17, y puede ser consultado en: https://docs.google.com/forms/d/e/1FAIpQLSfux6m1cU6Nf-69eiiMS28LjcSom38yqe2OmS-Jy4mXAgJVnA/ viewform

Para su validación (validez de contenido), se seleccionó inicialmente a un grupo de 56 jueces. Los criterios de selección fueron los siguientes: tener experiencia profesional en la utilización educativa de las TIC, tener experiencia en la Educación Especial, tener experiencia en la utilización de las TIC para personas discapacitadas, impartir docencia en TIC aplicadas a la educación o en educación especial, o trabajar en una institución relacionada con la educación especial. Utilizando para su selección el "Coeficiente de competencia experta" o "Coeficiente K" obtenido mediante la aplicación de la siguiente fórmula: $\mathrm{K}$ $=1 / 2(\mathrm{Kc}+\mathrm{Ka})$, donde $\mathrm{Kc}$ es el "Coeficiente de conocimiento" o información que tiene el experto acerca del tema o problema planteado; y Ka es el denominado "Coeficiente de argumentación" o fundamentación de los criterios de los expertos (Cabero y Barroso, 2013; Cabero, Fernández Batanero y Córdoba, 2016). En nuestro caso, el coeficiente $\mathrm{K}$ fue superior a 0,8 en 36 de los 56 expertos seleccionados inicialmente, que fueron los que al final se utilizaron para validar el cuestionario. Las estimaciones de los expertos se realizaron en sucesivas rondas, anónimas, al objeto de tratar de conseguir consenso, pero con la máxima autonomía por parte de los participantes (método Delphi). El nivel de fiabilidad (Alfa de Cronbach) obtenido tanto de manera general, como para cada una de las dimensiones fue el siguiente (Tabla 2).

Tabla 2. Alfa de Crombach general de la escala $y$ de sus diferentes dimensiones

\begin{tabular}{lc} 
Dimensión & Alfa de Crombach \\
\hline Total escala & $\mathbf{0 , 9 9 3}$ \\
Escala Aspectos generales & 0,967 \\
Escala Deficientes visuales & 0,986 \\
Escala Deficientes auditivos & 0,983 \\
Escala Deficientes motóricos & 0,982 \\
Escala Deficientes cognitivos & 0,979 \\
Escala Accesibilidad & 0,967 \\
\hline
\end{tabular}

Valores que de acuerdo con Mateo (2004), al estar ubicados en el intervalo 0.8 y 1 , pueden considerarse como "muy altas" y, en consecuencia, denotarían altos niveles de fiabilidad del instrumento elaborado.

\section{Resultados}

En la tabla 3 se presentan los valores medios alcanzados y sus desviaciones típicas obtenidas en los diferentes ítems que conformaban el cuestionario. 
Tabla 3. Valores medios y desviaciones típicas encontradas en los items del cuestionario.

\begin{tabular}{|c|c|c|}
\hline & Media & D. T. \\
\hline 1. Tengo conocimientos generales sobre las posibilidades que las TIC les ofrecen a las personas con discapacidad. & 4,02 & 2,64 \\
\hline 2. Conozco las dificultades que generan los diferentes tipos de discapacidad para el uso de las TIC. & 3,76 & 1,85 \\
\hline 3. Sabría seleccionar, TIC específicas en función de las características físicas, sensoriales y cognitivas de diferentes personas. & 3,37 & 2,36 \\
\hline $\begin{array}{l}\text { 4. Conozco diferentes recursos y documentos que se dedican específicamente al análisis de las posibilidades de las TIC } \\
\text { para las personas con diferentes tipos de discapacidad. }\end{array}$ & 1,56 & 1,99 \\
\hline 5. Conozco experiencias educativas de aplicación de las TIC para personas con diferentes tipos de discapacidad. & 2,70 & 2,75 \\
\hline 6. Conozco aplicaciones para móviles, en relación a los sujetos con necesidades educativas especiales. & 2,25 & 2,53 \\
\hline 7. Conozco las principales limitaciones que pueden condicionar el uso de las TIC por parte de alumnos con discapacidades. & 3,04 & 2,70 \\
\hline $\begin{array}{l}\text { 8. Me considero competente para localizar en la red materiales educativos para personas con necesidades educativas espe- } \\
\text { cíficas. }\end{array}$ & 3,13 & 2,78 \\
\hline $\begin{array}{l}\text { 9. En general, me siento preparado para ayudar al alumno con ciertas discapacidades en el uso de los apoyos técnicos y } \\
\text { utilización de las TIC. }\end{array}$ & 2,72 & 2,60 \\
\hline 10. Sé diseñar actividades con software educativos generalizados para el alumnado con necesidades educativas especiales. & 2,02 & 2,51 \\
\hline 11. Soy capaz de explicar las posibilidades que ofrece una máquina de escribir en sistema braille. & 2,58 & 2,88 \\
\hline 12. Se las posibilidades que ofrecen para los sujetos con discapacidad visual las máquinas lectoras Kurzweil. & 1,55 & 2,46 \\
\hline 13. Conozco las posibilidades que ofrecen las telelupas para los sujetos con discapacidad visual. & 2.27 & 2.79 \\
\hline 14. Reconozco diferentes programas informáticos específicamente producidos para personas con discapacidad visual. & 1,89 & 2,52 \\
\hline $\begin{array}{l}\text { 15. Conozco diferentes programas magnificadores de pantallas para facilitar el acceso a los sujetos con discapacidad visual } \\
\text { al ordenador. }\end{array}$ & 1,81 & 2,39 \\
\hline 16. Conozco diferentes softwares lectores de pantalla, como el JAWS, Tiflowin, etc... & 1,32 & 2,16 \\
\hline $\begin{array}{l}\text { 17. Sé realizar materiales didácticos en un procesador de texto eliminando los aspectos que dificultan su uso a personas con } \\
\text { discapacidad visual. }\end{array}$ & 2,12 & 2,56 \\
\hline $\begin{array}{l}\text { 18. Soy capaz de enumerar diferentes materiales tiflotecnológicos que permiten el acceso a las personas con discapacidad } \\
\text { visual al cálculo. }\end{array}$ & 1,44 & 2,22 \\
\hline 19. Conozco navegadores específicos para personas con discapacidad visual. & 1,29 & 2,06 \\
\hline 20. Conozco diferentes sitios web donde se pueden localizar recursos educativos para personas con discapacidad visual. & 1,75 & 2,34 \\
\hline $\begin{array}{l}\text { 21. Soy capaz de aplicar estrategias didácticas y adaptaciones curriculares apoyadas en TIC para facilitar la inclusión del } \\
\text { alumnado con discapacidad visual. }\end{array}$ & 2,28 & 2,45 \\
\hline 22. Conozco las posibilidades que las TIC le ofrecen al alumnado con limitaciones visuales. & 2,31 & 2,70 \\
\hline 23. Soy capaz de utilizar el lenguaje de signos. & 2,59 & 2,81 \\
\hline 24. Soy capaz de expresar mensajes de acuerdo a la lengua de signos. & 1,41 & 2,33 \\
\hline 25. Soy capaz de identificar diferentes recursos informáticos para la potenciación de la voz y del habla. & 1,85 & 2,41 \\
\hline $\begin{array}{l}\text { 26. Conozco diferentes programas informáticos educativos que sirven para la estimulación del desarrollo del lenguaje y la } \\
\text { adquisición y desarrollo de habilidades lingüísticas orales y escritas. }\end{array}$ & 1,89 & 2,20 \\
\hline $\begin{array}{l}\text { 27. Soy capaz de identificar diferentes sitios web donde se pueden localizar recursos educativos para personas con discapa- } \\
\text { cidad auditiva }\end{array}$ & 2,19 & 2,46 \\
\hline $\begin{array}{l}\text { 28. Soy capaz de aplicar estrategias didácticas apoyadas en TIC para facilitar la inclusión del alumnado con discapacidad } \\
\text { auditiva. }\end{array}$ & 1,75 & 2,29 \\
\hline $\begin{array}{l}\text { 29. Soy capaz de aplicar estrategias didácticas apoyadas en TIC para facilitar la inclusión de alumnos con discapacidad } \\
\text { auditiva. }\end{array}$ & 1,91 & 2,22 \\
\hline 30. Conozco las posibilidades que las TIC le ofrecen al alumnado con discapacidad auditiva. & 2,72 & 2,81 \\
\hline 31. Conozco diferentes programas de reeducación del habla. & 2,12 & 2,23 \\
\hline 32. Conozco diferentes tipos de teclados para personas con diferentes tipos de limitaciones en la movilidad. & 1,25 & 2,19 \\
\hline 33. Conozco los usos de los interruptores, conmutadores y punteros. & 1,17 & 2,09 \\
\hline 34. Conozco programas informáticos que controlan el ordenador con la voz & 2,22 & 1,99 \\
\hline $\begin{array}{l}\text { 35. Conozco los fundamentos de los sistemas alternativos de software aumentativos para facilitar la comunicación de las } \\
\text { personas con discapacidad motórica. }\end{array}$ & 1,97 & 2,32 \\
\hline 36. Localizo sitios web que contienen recursos educativos para personas con discapacidad motórica. & 1,48 & 2,01 \\
\hline $\begin{array}{l}\text { 37. Soy capaz de aplicar estrategias didácticas apoyadas en TIC para facilitar la inclusión del alumnado con limitaciones } \\
\text { motóricas. }\end{array}$ & 1,36 & 2,29 \\
\hline 38. Conozco las posibilidades que las TIC le ofrecen al alumnado con discapacidad motórica. & 2,00 & 2,54 \\
\hline
\end{tabular}




\begin{tabular}{|c|c|c|}
\hline 39. Puedo citar algunos programas educativos utilizados para la rehabilitación de habilidades cognitivas. & 2,01 & 2,39 \\
\hline $\begin{array}{l}\text { 40. Soy capaz de citar diferentes sitios web donde se pueden localizar recursos educativos para personas con discapacidad } \\
\text { cognitiva. }\end{array}$ & 1,24 & 1,01 \\
\hline 41. Sé utilizar software específico para realizar materiales para un teclado de concepto. & 1,14 & 1,99 \\
\hline $\begin{array}{l}\text { 42. Soy capaz de aplicar estrategias didácticas apoyadas en TIC para facilitar la inclusión del alumnado con discapacidad } \\
\text { cognitiva. }\end{array}$ & 1,99 & 2,35 \\
\hline 43. Soy capaz de realizar adaptaciones curriculares apoyadas en TIC para sujetos con discapacidad cognitiva. & 1,99 & 2,46 \\
\hline $\begin{array}{l}\text { 44. Soy capaz de describir las principales limitaciones que pueden contener los materiales multimedias para ser utilizados } \\
\text { con personas con discapacidad cognitiva. }\end{array}$ & 1,97 & 2,26 \\
\hline 45. Puedo localizar sitios web que contienen recursos educativos para personas con discapacidad cognitiva. & 2,66 & 2,73 \\
\hline 46. Conozco las posibilidades que las TIC le ofrecen al alumnado con discapacidad cognitiva. & 1,69 & 2,44 \\
\hline $\begin{array}{l}\text { 47. Conozco las posibilidades que nos ofrecen los sistemas operativos y los navegadores para modificar determinados } \\
\text { niveles de funcionamiento del programa (velocidad, tamaño de la letra, tipo de puntero,...) y hacer más accesible el } \\
\text { programa para personas con diferentes tipos de discapacidad. }\end{array}$ & 2,32 & 2,38 \\
\hline 48. Sé lo que son los test de accesibilidad para los sitios web. & 1,12 & 1,85 \\
\hline 49. Conozco las pautas generales de WAI/W3C que sirven para realizar los sitios web accesibles. & 1,15 & 2,00 \\
\hline 50. Soy capaz de crear páginas web con unos parámetros elevados de accesibilidad. & 1,93 & 2,56 \\
\hline $\begin{array}{l}\text { 51. Soy capaz de conseguir adaptar un equipo informático a las necesidades educativas de cualquier persona con discapa- } \\
\text { cidad. }\end{array}$ & 2,01 & 2,76 \\
\hline $\begin{array}{l}\text { 52. Conozco diferentes instituciones que están relacionadas con el estudio y la investigación de la accesibilidad de los sitios } \\
\text { web. }\end{array}$ & 1,66 & 1,98 \\
\hline 53. Soy capaz de citar diferentes test de accesibilidad & 1,27 & 2,02 \\
\hline
\end{tabular}

De la tabla anterior se desprende que en ninguno de los casos las puntuaciones medias obtenidos, alcanzaron el valor central de cinco. Al mismo tiempo, llama la atención las altas desviaciones típicas alcanzadas, que oscilaron entre 1,85 y 2,88. De ello, se desprende el poco conocimiento que el profesorado presenta respecto a la aplicación de las TIC para personas con algún tipo de discapacidad. Por un lado, las puntuaciones medias más elevadas se encontraron en los ítems: "Tengo conocimientos generales sobre las posibilidades que las TIC les ofrecen a las personas con discapacidad" $(4,02)$; "Conozco las dificultades que generan los diferentes tipos de discapacidad para el uso de las TIC" $(3,76)$; "Sabría seleccionar, TIC específicas en función de las características físicas, sensoriales y cognitivas de diferentes personas" $(3,37)$ $\mathrm{y}$ "Me considero competente para localizar en la redes materiales educativos para alumnos con necesidades específicas de apoyo educativo" $(3,13)$; "Me considero competente para localizar en la red materiales educativos para personas con necesidades educativas específicas" $(3,13)$.

Por otro lado, las puntuaciones más bajas se obtuvieron en los siguientes ítems: "Sé lo que son los test de accesibilidad para los sitios web" $(1,12)$; "Sé utilizar software específico para realizar materiales para un teclado de concepto" $(1,14)$; “Conozco las pautas generales de WAI/W3C que sirven para realizar los sitios web accesibles" $(1,15)$; y "Conozco los usos de los interruptores, conmutadores y punteros" $(1,17)$; "Soy capaz de citar diferentes sitios web donde se pueden localizar recursos educativos para personas con discapacidad cognitiva" $(1,24)$. También en estos casos las desviaciones típicas encontradas se situaron alrededor del valor " 2 ".

La puntuación media alcanzada en el total del instrumento fue de 2,02 con una desviación típica del 2,35 (Tabla 3). Estos valores nos indican, por un lado, que el profesorado presenta una baja autopercepción respecto a los conocimientos que poseen para la utilización de las TIC con sujetos con discapacidades, y por otro, la fuerte dispersión de las puntuaciones ofrecidas.
Tabla 4. Valores medios y desviaciones típicas encontradas en las dimensiones del cuestionario.

\begin{tabular}{lcc}
\hline Dimensiones & Media & D. tip. \\
General & 2,85 & 2,47 \\
Auditivos & 2.04 & 2,41 \\
Visuales & 1,88 & 2,46 \\
Motora & 1,63 & 2,20 \\
Cognitivos & 1,83 & 2,20 \\
Accesibilidad & 1,63 & 2,22 \\
Total & $\mathbf{1 , 9 7}$ & $\mathbf{2 , 3 2}$ \\
\hline
\end{tabular}

De las puntuaciones medias alcanzadas en la tabla anterior, podemos observar que el profesorado presenta un conocimiento escaso respecto a las aplicaciones de las TIC para personas con discapacidades, tanto de manera general como respecto a las discapacidades específicas (Visual, auditiva, motora y cognitiva), así como en relación a los conocimientos alusivos a la accesibilidad y al diseño universal.

Con respecto a conocer si el nivel de formación y conocimiento tecnológico, está determinado por variables como el género, años de experiencia o el tipo de centro donde ejerce su docencia, formulamos dos hipótesis, la nula (H0) hacía referencia a la no existencia de diferencias significativas desde un punto de vista estadístico con un riesgo alfa de equivocarnos del 0,05 o inferior y la alternativa (H1) a la existencia de tales diferencias en las mismas condiciones.

En relación con el nivel de formación y la variable "genero" se procedió a la realización de la prueba "U de Mann-Whitney" para muestras independientes (Siegel, 1976), tanto para el total del instrumento como para las diferentes dimensiones que lo conformaban. Por lo que se refiere a la puntuación alcanzada con el total del instrumento, en la tabla IV se presentan los valores obtenidos. 
Tabla 5. U de Mann-Whitney respecto a la formación para la utilización de las TIC para las personas discapacitadas y la variable sexo de los estudiantes (Nota: ${ }^{*}=0,05 y^{* *}=0,01$ ).

\begin{tabular}{cccc}
\hline Total del instrumento & U de Mann-Whitney & W de Wilcoxon & Nivel sig. \\
\hline general & 14617,500 & 97248,500 & $0,005\left(^{* *}\right)$ \\
\hline
\end{tabular}

Los valores obtenidos nos permiten rechazar la $\mathrm{H} 0 \mathrm{y}$ aceptar la $\mathrm{H} 1$ con un riesgo alfa de equivocarnos inferior al 0,01, y en consecuencia concluir que las autopercepciones que el profesorado tiene respecto a la formación que poseen para la aplicación de las TIC ante la discapacidad varían en función de género del profesorado. Puntuándose los profesores $(\mathrm{m}=2,31$; d. $\mathrm{t} .=2,37)$ de manera superior a las profesoras $(m=1,99 ; \mathrm{d}$. $\mathrm{t} .=2,13)$.

En relación con la existencia de diferencias significativas entre las percepciones del profesorado respecto a su formación para el uso de las TIC para personas con algún tipo de discapacidad y la variable años de experiencia (0 a 5 años; de 5 a 15 años; de 15 a 25 años; más de 25), aplicamos para ello el estadístico de Kruskal-Wallis (tabla IV).

Tabla 6. Puntuaciones alcanzadas por los alumnos en su formación y grado de magisterio cursado (Nota: ${ }^{*}=0,05 y^{* *}=0,01$ )

\begin{tabular}{lccc}
\hline Contraste & Kruskal-Wallis & Grados de libertad & Nivel sig. \\
\hline \multicolumn{4}{c}{ Total instrumento } \\
\hline \multicolumn{4}{c}{ Dimensiones } \\
general & 19,756 & 2 & $0,000\left(^{* *}\right)$ \\
visuales & 11,807 & 2 & $0,000\left(^{* *}\right)$ \\
auditivos & 21,842 & 2 & $0,000\left(^{* *}\right)$ \\
cognitivos & 10,730 & 2 & $0,004\left(^{* *}\right)$ \\
motora & 11,651 & 2 & $0,003\left(^{* *}\right)$ \\
accesibilidad & 16,522 & 2 & $0,000\left(^{* *}\right)$ \\
\hline
\end{tabular}

Los valores alcanzados nos permiten rechazar todas las hipótesis nulas (H0) formuladas y aceptar las alternativas (H1) con un riesgo alfa de equivocarnos inferior al 0,01, tanto en la puntuación total del cuestionario, como en las diferentes dimensiones que lo conforman. Luego podemos concluir, que los años de experiencia influyen en los conocimientos que afirman tener para la utilización de las TIC, tanto de forma general, para personas discapacitadas, como para personas con discapacidades visuales, auditivas, cognitivas o motora; así como su conocimiento respecto a la accesibilidad y el diseño universal.

Con el objetivo de poder conocer a favor de quiénes se daban las puntuaciones más altas, se presentan las puntuaciones medias alcanzadas en la globalidad del instrumento (Tabla 7).

Los resultados encontrados indican que es el profesorado con una experiencia de entre 0 y 5 años los que se consideran con más formación para aplicar las TIC a sujetos con diversidad funcional.

Para intentar saber si los resultados alcanzados variaban en función del tipo de centro donde ejercen su función docente, se procedió de nuevo a la aplicación del estadístico Kruskal-Wallis, para muestras independientes (tabla 8). En este caso los resultados encontrados no nos permiten rechazar la H0 formulada con un riesgo alfa de equivocarnos del 0,05 o inferior, y en consecuencia podemos concluir que no existen diferencias significativas en la formación que indican tener el profesorado para la aplicación de las TIC con personas con discapacidad, y la titularidad del centro educativo donde ejercen su docencia (Pública, privada-concertada y privada).

Tabla 7. Medias y desviaciones típicas alcanzadas en la globalidad del instrumento según los años de experiencia docente.

\begin{tabular}{cccccccc}
\multicolumn{2}{c}{ 0 a 5 años } & \multicolumn{2}{c}{ De 5 a 15 años } & \multicolumn{2}{c}{ De 15 a 25 años } & \multicolumn{2}{c}{ Más de 25 años } \\
\hline Media & Desv.t. & Media & Desv.t. & Media & Desv.t. & Media & Desv.t. \\
2.30 & 1,81 & 1,57 & 1,67 & 2,14 & 1,84 & 2,08 & 1,71 \\
\hline
\end{tabular}

Tabla 8. Puntuaciones alcanzadas por el profesorado en función del tipo de centro $\left(\right.$ Nota: $\left.{ }^{*}=0,05 y^{* *}=0,01\right)$.

\begin{tabular}{|c|c|c|c|}
\hline Contraste & Kruskal-Wallis & Grados de libertad & Nivel sig. \\
\hline \multicolumn{4}{|c|}{ Total instrumento } \\
\hline & 7,884 & 9 & 0,525 \\
\hline \multicolumn{4}{|c|}{ Dimensiones } \\
\hline general & 11,503 & 9 & 0,235 \\
\hline visuales & 7,340 & 9 & 0,601 \\
\hline auditivos & 7,221 & 9 & 0,692 \\
\hline cognitivos & 9,414 & 9 & 0,390 \\
\hline motora & 10,187 & 9 & 0,328 \\
\hline accesibilidad & 8,328 & 9 & 0,516 \\
\hline
\end{tabular}




\section{Conclusiones y discusión}

Los datos obtenidos a través de los cuestionarios aplicados a la muestra objeto de estudio nos ha permitido analizar los resultados y poder llegar a diversas conclusiones.

La primera que los datos han demostrado es la baja capacitación del profesorado de educación primaria respecto a la aplicación de las TIC para las personas discapacitadas. Tal baja formación se da independientemente del centro educativo donde ejercen su docencia.

La falta de formación constituye un obstáculo, sin lugar a dudas, para la inclusión del alumnado con diferente tipo de discapacidad, más siendo responsabilidad de la escuela el crear una escenografía, analógica y digital, que facilite la inclusión de la persona. Evidencia que concuerda con hallazgos de otros estudios (García García y López Azuaga, 2012; Marín, 2013; Cabero, Fernández Batanero y Córdoba, 2016).

Por otra parte, no descubrimos nada nuevo al señalar que el trato, humano y educativo, que recibe la persona discapacitada está fuertemente relacionado con la formación que posee el profesor respecto a los mismos; por ello es importante que estén capacitados para conocer las metodologías y las tecnologías adecuadas que favorezcan la inclusión (Roig, Ferrández, Rodríguez-Cano y Crespo, 2012; Tello y Cascales, 2015). Y ello, al menos en el caso de la tecnología, como se desprende de nuestros hallazgos, es bastante deficitaria. Tecnologías que no sólo sirven como elementos paliativos y minimizadores de la discapacidad (Cabero, Córdoba y Fernández Batanero, 2008), sino lo que es más importante para propiciar nuevas metodologías y estrategias didácticas y facilitar la comunicación e interacción de estas personas (Martínez, Raposo, y Añel, 2007; Córdoba, Cabero y Soto, 2012; Troncoso, Martínez, y Raposo, 2013; Pegalajar y Colmenero, 2014).

La segunda conclusión es la poca variabilidad de materiales que los maestros utilizan con estos estudiantes, como encuentran en su trabajo Ferrandis, Grau y Fortes (2010).

La tercera conclusión se dirije al ámbito de la accesibilidad y el diseño universal accesible. En este sentido, a pesar de la atención prestada por parte de la administración para favorecer la utilización de las TIC por las personas con algún tipo de discapacidad_(Toledo, Sánchez y Gutiérrez, 2013), la formación que señala el profesorado poseer es muy escasa. Hallazgo que se contrapone al de otros estudios donde se manifiesta que el profesorado cuando seleccionan aplicaciones y recursos TIC tienen muy encuenta la accesibilidad (Tello y Cascales, 2015).

A raíz de los resultados obtenidos, se precisa habilitar al docente para que adquiera estas competencias debido a que el rol docente tiene un efecto multiplicador en la educación, por tanto, las TIC, convertidas en herramientas de enseñanza aprendizaje, facilitan la creación de ambientes de aprendizaje que promuevan resultados integradores, en la medida que se convierten en elementos facilitadores de la inclusión educativa.

En esta línea, podemos afirmar que las TIC pueden ayudar al profesorado a la consecución de procesos de enseñanza y aprendizaje de mayor calidad, fomentando al mismo tiempo la inclusión educativa de "todo" el alumnado. Ahora bien, para ello, es necesario apostar por una mayor y mejor formación del profesorado de las distintas etapas educativas, en la utilización adecuada de las TIC. Aspecto que sólo es posible con la mejora de planes de formación continua acordes con la realidad de los centros, la realidad de los docentes y sobre todo, la realidad de los estudiantes.

\section{Limitaciones del estudio}

Como ya hemos señalado en trabajos anteriores, debemos decir que el carácter exploratorio de este estudio aconseja una interpretación prudente de los resultados, fundamentalmente a la hora de generalizar sus resultados a otros contextos. Pero el carácter inicial abre nuevos interrogantes de especial interés para la investigación en el campo de la TIC y la diversidad funcional (extender el estudio a todo el profesorado, diseñar y construir nuevos instrumentos que permitan un análisis más pormenorizado de las diferentes actitudes y competencias digitales del profesorado para responder a la inclusión educativa del alumnado,...).

\section{Referencias bibliográficas}

Alper, M. y Goggin, G. (2017). Digital technology and rights in the lives of children with disabilities. New Media $y$ Society. Disponible en http://journals.sagepub.com/doi/ full/10.1177/1461444816686323

Altinay A. y Altinay, Z. (2015). Examination on ICT integration into Special Education Schools for Developing Countries. TOJET: The Turkish Online Journal of Educational Technology, 14(3), 70-72

Bouck, CE, Doughty, TT, Flanagan, SM, Szwed, K., y Bassette, L. (2010). Es la más poderosa pluma? Utilizando Pentop computadoras para mejorar los estudiantes secundarios "escrito. Diario de Tecnología de la Educación Especial, 25, 33-47.

Cabero, J. Fernández Batanero, J. M. y Córdoba, M. (2016). Conocimiento de las TIC Aplicadas a las Personas con Discapacidades., Construcción de un Instrumento de Diagnóstico. En: Magis, Revista Internacional de Investigación en Educación, Vol. 8. Núm. 17, 157-176.

Cabero, J. y Barroso, J. (2013). La utilización del juicio de experto para la evaluación de TIC: el coeficiente de competencia experta. Bordón, 65(2) 25-38.

Cabero, J. y Guerra, S. (2011). La alfabetización y formación en medios de comunicación en la formación inicial del profesorado. Educación XX1, 14(1) 89-115.

Cabero, J., Córdoba, M. y Fernández Batanero, J.M. (2008). Las TIC para la igualdad. Sevilla: Eduforma.

Córdoba, M. Cabero, J. y Soto, F.J. (2012). Buenas prácticas de aplicación de las TIC para la igualdad. Sevilla: Eduforma.

Ferrandis, M.V., Grau, C. y Fortes, M.C. (2010). El profesorado y la atención a la diversidad en la ESO. Revista de Educación Inclusiva, 3(2), 11-28.

García, M. y López, R. (2012). Explorando desde una perspectiva inclusiva el uso de las TIC para atender a la diversidad. Profesorado. Revista de curriculum y formación del profesorado, 16(1). Recuperado de http://www.ugr.es/local/recfpro/rev161COL4.pdf

Ghaleb, A. (2014). Assistive technology in special education and the universal design for learning. TOJET: the Turkish online Journal of Educational Technology, 13 (2), 18-23.

Homero, G., Tejedor, F. J. y Calvo, M. I. (2017). Meta-análisis sobre el efecto del software educativo en alumnos con necesidades educativas especiales. Revista de Investigación Educativa, 35(1), 35-52.

Istenic, A. y Bagon, S. (2014). ICT-supported learning for inclusion of people with special needs: Review of seven educational technology journals, 1970-2011, British Journal of Educational Technology, 45(2), 202-230.

Khetarpal, A. (2015). Information and Communication Technology (ICT) and Disability. Review of market integration, 6(1), 96-103. 
Liu, S. H. (2011). Un modelo multivariado de factores que influyen Uso de Tecnología por futuros profesores durante la Práctica Enseñanza. Tecnología para la Educación y Sociedad, 15 (4), 137-149.

Marín, V. (coord.) (2013), Desarrollando la competencia digital desde la educación inclusiva, Mataró, Da Vinci.

Martínez, E., Raposo, M. y Añel, E. (2007): La potencialidad de los materiales en la promoción de la escuela inclusiva. Revista Iberoamericana de Evaluación Educativa, 5(3) 47-63.

Mateo, J. (2004). La investigación ex post-facto. En R. Bisquerra-Alzina (coord.). Metodología de la investigación educativa (pp. 195-230). Madrid: La Muralla.

OCDE (2012). Equity and Quality in Education: Supporting Disadvantaged Students and Schools. Bruselas: OCDE.

OEI (2010). 2021: Metas educativas. La educación que queremos para la generación de los bicentenarios, Madrid, OEI.

Ortíz, A., Almazán, L., Peñaherrera, M. y Cachón J. (2014). Formación en TIC de futuros maestros desde el análisis de la práctica en la Universidad de Jaén. Pixel-Bit. Revista de Medios y Educación, 44, 127-142.

Patton, CM, y Roschelle, J. (2008, 7 de mayo). ¿Por qué el mejor plan de estudios de matemáticas no será un libro de texto. Educación Semana, 24-32.

Pegalajar, M.C. y Colmenero, M.J. (2014). Estudio piloto sobre el uso de las redes sociales en jóvenes con discapacidad intelectual. EDUTEC, Revista Electrónica de Tecnología Educativa, 48. Disponible en http://edutec.rediris.es/Revelec2/Revelec48/ n48 Pegalajar Colmenero.html

Prendes, M.P. y Gutiérrez, I. (2013). Competencias tecnológicas del profesorado en las universidades españolas. Revista de Educación, 361. 196-222.

Rangel, P. y Peñalosa, E. (2013). Alfabetización digital en docentes de educación superior: construcción y prueba empírica de un instrumento de evaluación. Pixel-Bit. Revista de Medios y Educación, 43, 9-23.

Roig, R.; Ferrández, S.; Rodríguez-Cano, C. y Crespo, M. (2012). El uso de las TIC en el aula de Educación Especial: percepción de los maestros. En J. Navarro, M. T. Fernández, F. J. Soto y F. Tortosa (coords.). Respuestas flexibles en contextos educativos diversos. Murcia: Consejería de Educación, Formación y Empleo. Disponible en http://diversidad. murciaeduca.es/publicaciones/dea2012/docs/ rroig.pdf

Rosario, H. y Vásquez, L. (2012). Formación del docente universitario en el uso de tic. Caso de las universidades públicas y privadas. (U. de Carabobo y U. Metropolitana). Pixel-Bit. Revista de Medios y Educación, 41, 163-171.
Suárez, J. y otros (2013). Las competencias del profesorado en TIC: estructura básica. Educación XX1. 16.1, 39-62.

Suriá R., Martínez, D. y Ordoñez, T. (2010). TIC, docencia y discapacidad: ¿se sienten preparados los docentes para apoyar al alumnado discapacitado en el uso de las TIC en las aulas? en "25 años de integración escolar en España. Tecnología e inclusión en el ámbito educativo, laboral y comunitario", Murcia, Consejería de Educación, Formación y Empleo, pp. 1-5.

Tello, I. y Cascales, A. (2015). Las TIC y las necesidades específicas de apoyo educativo: análisis de las competencias tic en los docentes. RIED, 18(2), 355-383.

Terigi, F. (2013). VIII Foro Latinoamericano de Educación: saberes docentes: qué debe saber un docente y por qué. Buenos Aires: Santillana.

Toledo, P. (2013). Las tecnologías de la información, la comunicación y la inclusión educativa. En: Nuevos escenarios digitales. Las Tecnologías de la Información y la Comunicación aplicadas a la formación y desarrollo curricular (411-427). Madrid: Pirámide.

Toledo, P., Sánchez, J.M. y Gutiérrez, J.J. (2013). Evolución de la accesibilidad web en las Universidades Andaluzas. Pixel-Bit. Revista de Medios y Educación, 43, 65-83.

Torres, L. (2007). La accesibilidad de las TIC, en Cabero, J. Córdoba, M. y Fernández Batanero, J.M. (2007). Las TIC para la igualdad. Sevilla: Eduforma, 197-218.

Troncoso, A.B., Martínez, M.E. y Raposo, M. (2013). La inclusión del alumno con discapacidad intelectual a partir del uso de blogs: una experiencia educativa innovadora. Revista Latinoamericana de Inclusión Educativa, 7(2), 195-211. Disponible en http://www.rinace.net/rlei/numeros/vol7-num2/art11.pdf

Valdivieso, T. S. y González, M. A. (2016). Competencia digital docente: ¿Dónde estamos? Perfil del docente de educación primaria y secundaria. El caso de Ecuador. Píxel-Bit. Revista de Medios y Educación, 49, 57-73. Disponible en http://dx.doi. org/10.12795/pixelbit.2016.i49.04

Vladimirovna, S. y Sergeevna,O. (2015). Features of the Information and Communication Technology Application by the Subjects of Special Education. International Education Studies; 8 (6), doi:10.5539/ies.v8n6p162, recuperado de http://www. ccsenet.org/journal/index.php/ies/article/view/49385;

Wallace, T. y Georgina, D. (2014). Preparing special education teachers to use educational tecnology to enhance student learning. 11th International Conference on Cognition and Exploratory Learning in Digital Age.

Yusof, A. M., Gnanamalar, E., Daniel, S., Low, W. y Aziz, K. (2014). Teachers' perception of mobile edutainment for special needs learners: the Malaysian case. International Journal of Inclusive Education, 18(2). 